\title{
Prospective comparison of sinonasal outcomes after microscopic sublabial or endoscopic endonasal transsphenoidal surgery for nonfunctioning pituitary adenomas
}

\author{
Carrie L. Pledger, MA, ${ }^{1}$ Mohamed A. Elzoghby, MD, ${ }^{2}$ Edward H. Oldfield, MD, ${ }^{1}$ \\ Spencer C. Payne, MD, ${ }^{3}$ and John A. Jane Jr., MD ${ }^{1}$
}

Departments of ${ }^{1}$ Neurosurgery and ${ }^{3}$ Otolaryngology-Head and Neck Surgery, University of Virginia, Charlottesville, Virginia; and 2Department of Neurosurgery, Ain Shams University School of Medicine, Cairo, Egypt

OBJECTIVE Both endoscopic and microscopic transsphenoidal approaches are accepted techniques for the resection of pituitary adenomas. Although studies have explored patient outcomes for each technique individually, none have prospectively compared sinonasal and quality of life outcomes in a concurrent series of patients at the same institution, as has been done in the present study.

METHODS Patients with nonfunctioning adenomas undergoing transsphenoidal surgery were assessed for sinonasal function, quality of life, and pain using the Sino-Nasal Outcome Test-20 (SNOT-20), the short form of the Nasal Obstruction Symptom Evaluation (NOSE) instrument, the SF-36, and a headache scale. Eighty-two patients undergoing either endoscopic (47 patients) or microscopic (35 patients) surgery were surveyed preoperatively and at 24-48 hours, 2 weeks, 4 weeks, 8 weeks, and 1 year after surgery.

RESULTS Patients who underwent endoscopic and microscopic transsphenoidal surgery experienced a similar recovery pattern, showing an initial increase in symptoms during the first 2 weeks, followed by a return to baseline by 4 weeks and improvement beyond baseline functioning by 8 weeks. Patients who underwent endoscopic surgery experienced better sinonasal outcomes at 24-48 hours (SNOT total $p=0.015$, SNOT rhinologic subscale [ssRhino] $p<0.001$ ), 2 weeks (NOSE $p=0.013$ ), and 8 weeks (SNOT total $p=0.032$ and SNOT ssRhino $p=0.035$ ). By 1 year after surgery, no significant differences in sinonasal outcomes were observed between the 2 groups. Headache scales at 1 year improved in all dimensions except duration for both groups (total result 73\%, $p=0.004$; severity 46\%, $p<0.001$; frequency $53 \%, p<0.001$ ), with $80 \%$ of either microscopic or endoscopic patients experiencing improvement or resolution of headache symptoms. Endoscopic and microscopic patients experienced reduced vitality preoperatively compared with US population norms and remained low postoperatively. By 8 weeks after surgery, both groups experienced significant improvements in mental health $(13 \%, p=0.005)$ and vitality $(15 \%, p=0.037)$. By 1 year after surgery, patients improved significantly in mental health $(14 \%, p=0.03)$, role physical $(14 \%, p=0.036)$, social functioning $(16 \%, p=0.009)$, vitality $(22 \%, p=0.002)$, and SF-36 total $(10 \%, p=0.024)$ as compared with preoperative measures. There were no significant differences at any time point between the 2 groups for the total SF-36 or for any of the 8 subscales.

CONCLUSIONS Patients who underwent either an endoscopic or a microscopic approach experienced the greatest nasal symptoms at 2 weeks postoperatively and exhibited similar time courses of recovery in nasal, headache, and quality of life assessments. Although patients who underwent endoscopic surgery experienced significantly fewer nasal symptoms during the first 8 weeks, by 1 year after surgery, there were no significant differences between the 2 groups. http://thejns.org/doi/abs/10.3171/2015.6.JNS142695

KEY WORDS transsphenoidal; outcomes; pituitary surgery 
$\mathrm{T}$ HE transsphenoidal resection of pituitary adenomas is performed using either an endoscope or a microscope. ${ }^{2,3}$ Advantages of the endoscopic approach include an improved view of the anatomy, a wider view inside the sella, and a greater working area. By contrast, the microscopic approach allows for high-resolution imaging and improved visualization of extremely small microadenomas. ${ }^{23}$ Use of the endoscope is thought to provide the patient with fewer postoperative symptoms and less pain as well as a quicker recovery. While some authors have argued that the endoscope is a less invasive technique, others have reported that the endoscopic approach disrupts a greater amount of normal anatomy and requires more extensive exposure than the microscopic technique. ${ }^{8,15,31}$

Few studies have investigated the differences in patients' experiences with either microscopic or endoscopic surgery, and none have done so prospectively in a concurrent series of patients treated with the same postoperative protocol. We sought to determine if the specific approach to the pituitary, endoscopic versus microscopic, imparted any differences in patient-related quality of life (QOL), including sinonasal symptoms and their rate of recovery.

To address these questions, we designed and executed a prospective study of patients' self-reported symptoms and QOL before and at several time points after surgery. Interpretations of previous studies are confounded by the inclusion of a wide variety of diagnoses, including all types of pituitary adenomas, craniopharyngiomas, Rathke's cleft cysts, and other skull-based diagnoses. To eliminate this issue, we chose to limit the current study to patients with nonfunctioning adenomas (NFAs). Furthermore, while prior studies have assessed patient QOL at different time points after endoscopic surgery,,$^{8,20,21,28}$ no other studies to our knowledge have examined the differences in QOL and sinonasal outcomes of microscopic and endoscopic surgery for defined time points in a concurrent series of patients at the same institution.

Outcomes-based research is important for patient care and analyses by insurance companies. As a result of outcomes-based research, patients can better understand what to expect and physicians can more accurately counsel the patients before and after surgery. Our study aims to determine the physical and mental differences, if any, experienced by patients undergoing either microscopic or endoscopic surgery for an NFA during the immediate perioperative (24-48 hours after surgery), short-term (2, 4 , and 8 weeks), and long-term (1 year) recovery stages.

\section{Methods}

\section{Patient Recruitment}

Patient recruitment was performed at the Neuroendocrine Clinic at the University of Virginia under a protocol approved by the Institutional Review Board for Health Sciences Research (IRB-HSR) at the University of Virginia.

All patients over the age of 18 years with a diagnosis of NFA and a scheduled date for transsphenoidal surgery between March 2009 and June 2011 were eligible for inclusion. Patients were excluded if a combination of microscopic and endoscopic techniques was used in the surgical procedure. Eligible patients were asked to complete 4 preoperative questionnaires concerning their health: SinoNasal Outcome Test-20 (SNOT-20), ${ }^{16}$ Nasal Obstruction Symptom Evaluation (NOSE), ${ }^{17} \mathrm{SF}-36,{ }^{18}$ and a headache scale. ${ }^{11}$ Patients were then given follow-up surveys for the SNOT-20 and NOSE postoperatively on Day 1 or 2, 2 weeks, 4 weeks, 8 weeks, and 1 year. The SF-36 surveys were distributed at the 8-week and 1-year time points.

Each patient who returned a preoperative packet was enrolled in the study and followed up postoperatively. Patients who did not return a preoperative questionnaire were considered to have declined participation in the study. Surveys for postoperative Days 1 and 2 were presented in paper form to the patients while in the hospital. Surveys for 2 and 4 weeks after surgery were delivered to the patient via either email or phone by the same employee. Surveys at 8 weeks and 1 year after surgery were performed when patients returned to the pituitary clinic for their first postoperative visit. Patients who did not return to clinic or failed to complete the survey were mailed the surveys with a self-addressed return envelope.

Only patients who returned a preoperative survey and at least 1 follow-up survey were included in the data to be analyzed. Missing data due to noncompliance, missed appointments, or any other reason were left blank for that specific analysis time point. The entire study was analyzed using a cross-sectional approach at each time point.

\section{Surveys}

The SNOT-20 is a validated 20-question assessment that measures nasal functioning and related outcomes. ${ }^{26}$ The SNOT-20 summary scores are based on a 0 to 5 scaling system, where 0 reflects "no problems" and 5 indicates a "problem as bad as it can be." The total SNOT-20 score was used to assess each patient's overall symptoms. Given the range of questions presented in the SNOT-20 survey, recent literature has proposed the use of subscales to delineate the specific factors involved when reporting the results of SNOT-20.,26 The subscale constructs of the SNOT-20 address rhinologic, psychological, ear and facial, and sleep symptoms (ssRhino, ssPsych, ssEar/Face, ssSleep, respectively). The NOSE short form is a 4-question scale representative of nasal function. ${ }^{27}$ Scores were based on a 0 to 5 scaling system. Higher scores represent poorer function for both surveys.

A headache score was also calculated for each patient using the formula described by Levy et al. ${ }^{18}$ Headache was determined using 3 factors: greatest severity for peak attack (range $0=$ no pain to $10=$ worst pain), duration in number of hours per day (0 to 24 hours), and frequency in number of days per week (0 to 7 days). A final score was computed by multiplying the 3 factors (severity $\times$ duration $x$ frequency) resulting in a minimum score of 0 through a maximum score of 1680 (10 severity $\times 24$ hours $\times 7$ days). These scores were attained preoperatively and at 2 weeks, 4 weeks, 8 weeks, and 1 year after surgery. The headache score was not assessed while the patient was in the hospital because the average length of stay was 2-3 days and the headache scale required a frequency assessment based on 7 days per week.

The SF-36 assessment of QOL is based on a 0 to 100 scaling system. The SF-36 contains 36 nonspecific, vali- 
dated health questions and addresses 8 domains of health: physical functioning, role limitations due to physical functioning, emotional functioning, role limitations due to emotional functioning, bodily pain, social functioning, general health, and vitality (energy and fatigue). ${ }^{31}$ Higher scores on the SF-36 indicate higher health satisfaction.

\section{Surgical Techniques}

All surgical procedures were conducted at the University of Virginia Health System. The endoscopic technique was performed by a single surgeon (J.A.J.) using a binarial 3-hand technique with lateralization of the middle turbinates, limited posterior septectomy, and resection of the inferior one-third of the superior turbinates bilaterally. ${ }^{15}$ The microscopic technique was performed by a single surgeon (E.H.O.) using a sublabial submucosal technique, as previously described. ${ }^{24}$ Regardless of the procedure performed, patient nasal care was uniform postoperatively. Patients were provided with oxymetazoline twice daily for the first 3 days postoperatively and hourly saline nasal sprays during waking hours for 2 weeks. Thereafter, patients were instructed to use saline nasal sprays every 3-4 hours until the follow-up at 8 weeks. Patients were also instructed to use a buffered saline nasal rinse 3 times a day starting on postoperative Day 3 for the first 2 weeks. After 2 weeks patients were to use the nasal rinse at least twice daily until the follow-up at 8 weeks. No patient underwent planned outpatient rhinologic follow-up for debridement or was provided prophylactic antibiotics.

\section{Statistical Analysis}

We analyzed the data using standard software (Excel, SPSS Inc.). Student t-test was used to compare the SNOT, NOSE, and SF-36 scores between patients who underwent the endoscopic procedure and those who underwent the microscopic technique. Sample size varied at each time point because patients did not complete all of the surveys; therefore, a cross-sectional approach was used to compare the individual time point differences. The results for each time period were shown as the statistical difference between technique groups, and the value of $\mathrm{N}$, the number of surveys completed, was noted for each time period (Table 1). A p value $<0.05$ was considered statistically significant.
TABLE 2. Characteristics of patients who underwent transsphenoidal surgery for a nonfunctioning pituitary tumor utilizing either the endoscopic or microscopic technique

\begin{tabular}{ccccc}
\hline Technique & $\begin{array}{c}\text { No. of } \\
\text { Patients }\end{array}$ & Sex (M:F) & $\begin{array}{c}\text { Mean Age in } \\
\text { Yrs (range) }\end{array}$ & $\begin{array}{c}\text { Mean Tumor } \\
\text { Size }(\mathrm{mm})\end{array}$ \\
\hline Endo & 47 & $23: 24$ & $52(32.5-79.5)$ & 23.6 \\
\hline Micro & 35 & $17: 18$ & $54(27-74)$ & 23.5 \\
\hline
\end{tabular}

\section{Results}

One hundred fifty-five consecutive patients scheduled for resection of a nonfunctioning pituitary tumor were contacted to enroll in the study before their surgery. Eighty-two patients completed the preoperative surveys, of whom 47 had been scheduled for surgery using an endoscopic technique and 35 had been scheduled for surgery utilizing a microscopic technique. Patients who underwent microscopic and endoscopic techniques were similar in age, sex, and average tumor size (Table 2). There were no postoperative cerebrospinal fluid (CSF) leaks in either cohort.

\section{Scale Results}

The general trend of both the SNOT-20 and NOSE scores was similar after either endoscopic or microscopic surgery; patients reached a symptomatic peak at 2 weeks and gradually returned to baseline or better between 8 weeks and 1 year postoperatively (Figs. 1-3). Headache scores steadily improved over the postoperative course (Figs. 4 and 5). Quality of life, as measured by the SF-36 (total), improved at both 8 weeks and 1 year postoperatively (Fig. 6). On average all patients returned to baseline levels by 1 year after surgery or showed improvement beyond their baseline condition.

\section{SNOT-20 Results}

Seventy-nine patients completed the SNOT surveys (46 endoscopic and 33 microscopic). Microscopic patients experienced a significant increase in rhinologic subscale (ssRhino) symptoms within the first 24-48 hours after surgery $(\mathrm{p}=0.001)$ compared with before surgery (Fig. 2). By 2 weeks postoperatively, microscopic patients ex-

TABLE 1. Sample sizes at each time point*

\begin{tabular}{cccccccc}
\hline \multirow{2}{*}{ Assessment Scale } & \multicolumn{5}{c}{ No. of Completed Surveys per Time Point } \\
\cline { 3 - 8 } & Technique & Baseline & 24-48 Hrs & 2 Wks & 4 Wks & 8 Wks & 1 Yr \\
\hline \multirow{2}{*}{ SNOT-22 } & Endo & 46 & 40 & 27 & 23 & 31 & 31 \\
\cline { 2 - 8 } & Micro & 33 & 26 & 19 & 19 & 30 & 18 \\
\hline \multirow{2}{*}{ NOSE } & Endo & 45 & 34 & 27 & 23 & 30 & 31 \\
\cline { 2 - 8 } & Micro & 33 & 24 & 19 & 19 & 30 & 18 \\
\hline \multirow{2}{*}{ Headache } & Endo & 30 & - & 18 & 14 & 18 & 20 \\
\cline { 2 - 8 } & Micro & 24 & - & 16 & 17 & 24 & 15 \\
\hline \multirow{2}{*}{ SF-36 } & Endo & 40 & - & - & - & 31 & 34 \\
& Micro & 34 & - & - & - & 31 & 25 \\
\hline
\end{tabular}

Endo = endoscopic surgery; micro = microscopic surgery; - = time points where no surveys were given.

* Sample sizes on a per time point basis used for statistical analysis varied depending on survey completion by patients. 


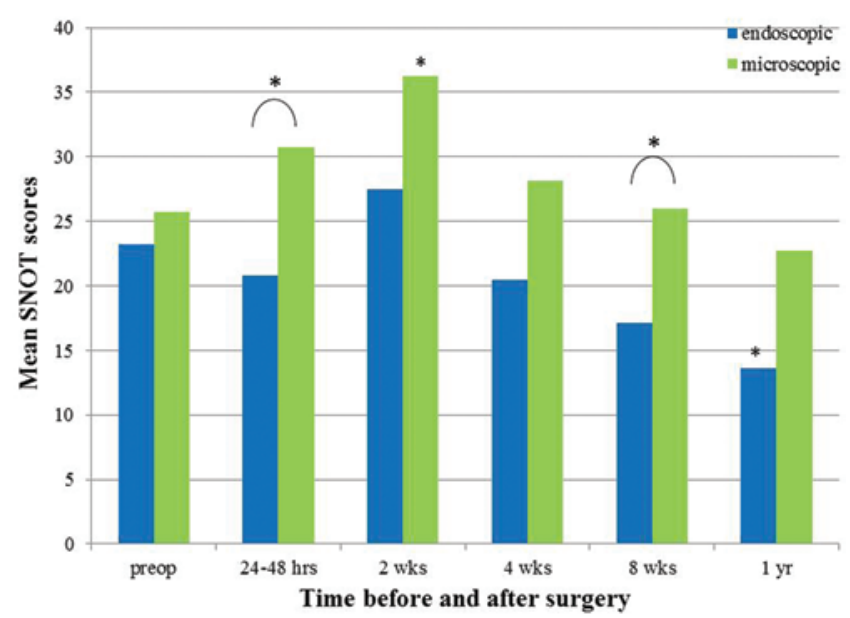

FIG. 1. Mean SNOT scores in microscopic and endoscopic patients. Bar graph shows that patients who undergo endoscopic surgery experience better sinonasal outcomes than those who undergo microscopic surgery at $24-48$ hours (SNOT total mean microscopic $=30.73$ vs mean endoscopic $=20.83, p=0.015$ ) and 8 weeks (SNOT total mean microscopic $=25.97$ vs mean endoscopic $=17.13, p=0.032$ ). Compared with baseline measures, microscopic patients have a significant increase in SNOT scores at 2 weeks $(p=0.15)$. Endoscopic patients have a significant improvement in SNOT scores by 1 year $(p=0.016)$ compared with baseline. Asterisk indicates a significant $(p<0.05)$ difference in mean SNOT scores; $\cap$ indicates a difference in mean SNOT subscale scores between microscopic and endoscopic groups. Figure is available in color online only.

perienced an increase in rhinologic symptoms $(\mathrm{p}=0.001)$ and the SNOT total score $(\mathrm{p}=0.015)$ compared with their preoperative condition (Figs. 1 and 2). By 4 weeks postoperatively and thereafter, these patients returned to their preoperative rhinologic SNOT status.

Conversely, endoscopic patients experienced a slight improvement in SNOT scores during the perioperative period, but this did not reach statistical significance (Fig. 1). By 2 weeks postoperatively, endoscopic patients experienced an increase in rhinologic symptoms compared with their baseline measures ( $\mathrm{p}=0.001$; Fig. 2). By 4 weeks and 8 weeks postoperatively, patients returned to their preoperative rhinologic and total SNOT status. By 1 year after surgery, the endoscopic group continued to improve significantly better than baseline both in total SNOT score ( $p=0.016$; Fig. 1) and on the psychological subscale (ssPsych; $p=0.007$ ). Baseline preoperative endoscopic and microscopic SNOT scores (Fig. 1) were not significantly different (mean endoscopic score $=23.2$, mean microscopic score $=25.7$ ). The most significant difference between the surgical techniques was seen on the SNOT total score within the first 24-48 hours after surgery (mean microscopic $=30.73$ vs mean endoscopic $=$ $20.83, p=0.015$; Fig. 1). At this time point the ssEar/Face $(\mathrm{p}=0.018)$ and ssRhino ( $\mathrm{p}<0.001$; Fig. 2$)$ contributed most significantly to the SNOT total score differences seen between the 2 groups. No significant differences were notable between the 2 techniques at 2 or 4 weeks after transsphenoidal resection. By 8 weeks postoperatively, rhinologic function and total SNOT score had improved significantly more in the endoscopic patients than

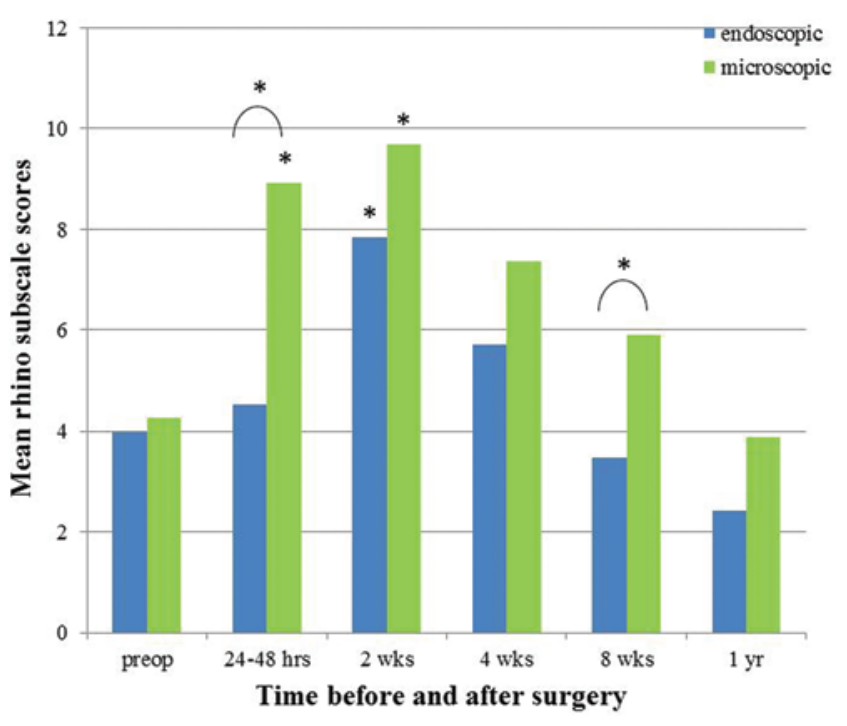

FIG. 2. Mean rhinologic (rhino) SNOT subscale score in microscopic and endoscopic patients. Bar graph shows rhinologic symptoms measured by a subscale of SNOT (ssRhino). Patients who undergo endoscopic surgery have significantly fewer rhinologic symptoms at 24-48 hours $($ mean microscopic $=8.92$ vs mean endoscopic $=4.27, p<0.001$ ) and 8 weeks (mean microscopic $=5.90$ vs mean endoscopic $=3.48, p=$ $0.035)$ postoperatively than patients who undergo microscopic surgery. Compared with baseline measures, microscopic patients experience a significant increase in rhinologic subscale symptoms within the first 24-48 hours $(p=0.001)$ and 2 weeks $(p=0.001)$ postoperatively. Endoscopic patients experience an increase in rhinologic symptoms at 2 weeks compared with their baseline measures (endoscopic $p=0.001$ ). Asterisk indicates a significance of $p<0.05$; $\cap$ indicates a difference in mean rhinologic SNOT subscale scores between microscopic and endoscopic groups. Figure is available in color online only.

in the microscopic patients (ssRhino mean microscopic $=5.90$ vs mean endoscopic $=3.48, \mathrm{p}=0.035$; SNOT-20 total mean microscopic $=25.97$ vs mean endoscopic $=$ $17.13, \mathrm{p}=0.032$ ), but the difference between the groups lost significance by 1 year after surgery. There were no significant differences on either of the QOL subscales (psychological and sleep function), and no significant difference was found between techniques for any construct at any of the other time points.

\section{NOSE Results}

Seventy-eight patients completed the NOSE surveys (45 endoscopic and 33 microscopic). When analyzed individually, each surgical approach was associated with a significant decrease in nasal function 24-48 hours after surgery (endoscopic $\mathrm{p}=0.014$ and microscopic $\mathrm{p}<$ 0.0001 ) (Fig. 3). Although the endoscopic group returned to baseline NOSE scores by 2 weeks postoperatively, the microscopic group continued to have decreased nasal function at 2 weeks $(p<0.0001)$. Whereas microscopic patients returned to baseline scores at 4 weeks and did not improve further, endoscopic patients experienced significant improvements in NOSE scores beyond baseline at both 8 weeks $(p=0.027)$ and 1 year $(p=0.009)$.

Baseline preoperative endoscopic and microscopic NOSE scores (Fig. 3) were not significantly different from one another (mean endoscopic score $=1.01 \mathrm{vs}$ mean mi- 


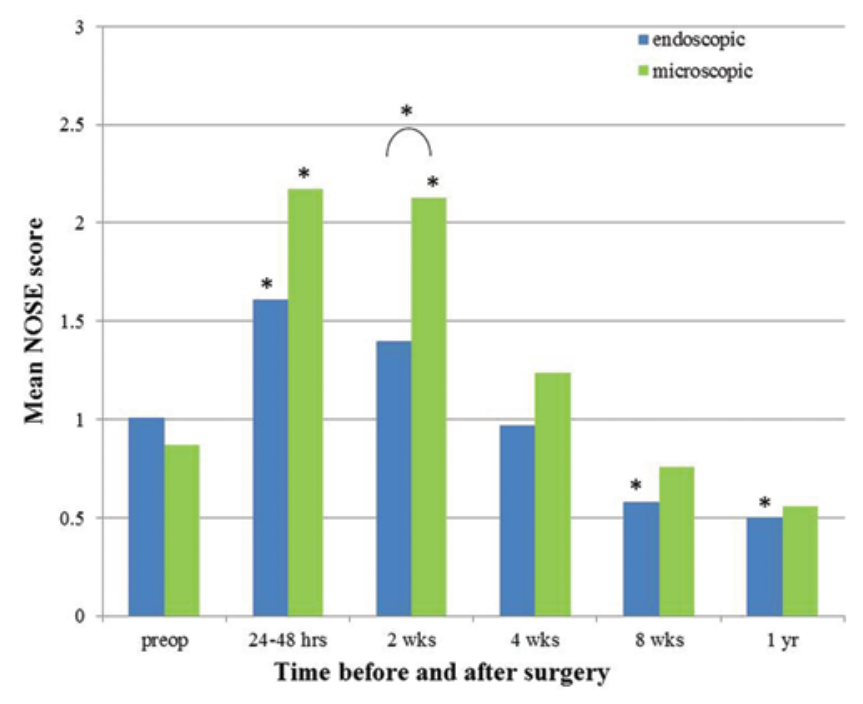

FIG. 3. Bar graph represents the NOSE data in microscopic and endoscopic patients. Patients who undergo endoscopic surgery experience better NOSE outcomes at 2 weeks (mean endoscopic $=1.4$ vs mean microscopic $=2.13, p=0.029$ ) than those who undergo microscopic surgery. Compared with baseline measures, microscopic patients have an increase in NOSE scores at 24-48 hours $(p<0.001)$ and 2 weeks ( $p$ $=0.001$ ). Endoscopic patients have an increase in NOSE scores 24-48 hours after surgery $(p=0.014)$ followed by improvement, which reaches significance by 8 weeks $(p=0.027)$ and 1 year $(p=0.009)$. Asterisk indicates a significant $(p<0.05)$ difference in mean NOSE scores in microscopic versus endoscopic groups; $\cap$ indicates a difference in mean NOSE subscale scores between microscopic and endoscopic groups. Figure is available in color online only.

croscopic score $=0.87$ ). The only significant difference in the total NOSE score between the surgical techniques occurred at the 2 -week time period $(\mathrm{p}=0.029)$. At 2 weeks, patients undergoing the endoscopic approach had significantly better average NOSE scores (mean endoscopic =
1.4 vs mean microscopic $=2.13, \mathrm{p}=0.029$ ). Two important contributors to the differences seen at 2 weeks were the differences in 2 factors: 1) "unable to get enough air through my nose during exercise" (mean endoscopic = 0.67 vs mean microscopic $=1.68, p=0.017$ ) and 2) "nasal congestion/stuffiness" (mean endoscopic $=2.04$ vs mean microscopic $=2.79, \mathrm{p}=0.040)$. There was also a significant difference in "trouble sleeping" during the perioperative period (mean endoscopic $=1.42$ vs mean microscopic $=2.13, \mathrm{p}=0.043)$.

\section{Headache Results}

Seventy-seven patients completed the headache scales (45 endoscopic and 32 microscopic), and $70 \%$ of these patients (30 endoscopic and 24 microscopic) had preoperative headaches. Microscopic patients had no significant improvements at 2, 4, or 8 weeks after surgery compared with the preoperative state. However, by 1 year after surgery, microscopic patients improved significantly in all areas except headache duration (frequency 56\%, $\mathrm{p}=0.001$; severity $35 \%, \mathrm{p}=0.033$; total headache result $75 \%, \mathrm{p}=$ 0.031).

Endoscopic patients also had no significant improvements at 2 or 4 weeks after surgery compared with the preoperative state. Endoscopic patients improved significantly in headache severity at 8 weeks postoperatively $(44 \%, \mathrm{p}=0.017)$ and improved significantly in frequency $(51 \%, \mathrm{p}=0.005)$ and severity $(56 \%, \mathrm{p}=0.001)$ by 1 year after surgery compared with baseline.

We then compared the number of microscopic (Fig. 4 left) or endoscopic (Fig. 4 right) patients whose headache scores increased, decreased, or remained stable at each time point compared with baseline. We determined that $45 \%$ of patients experienced a worsening of headaches by 2 weeks postoperatively, while $51 \%$ experienced improvement in headaches as early as 2 weeks postoperatively in either group. Both groups continued to improve from 4

\section{Endoscopic patients}

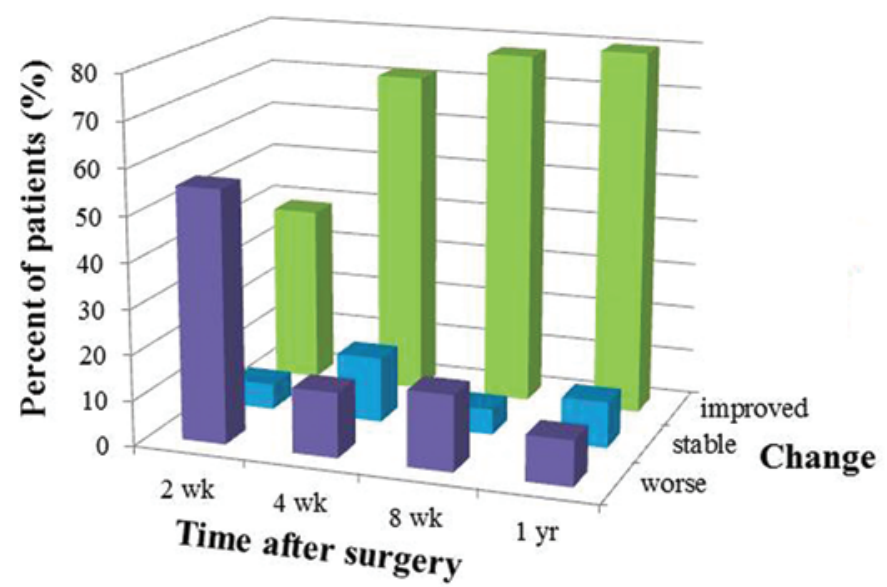

\section{Microscopic patients}

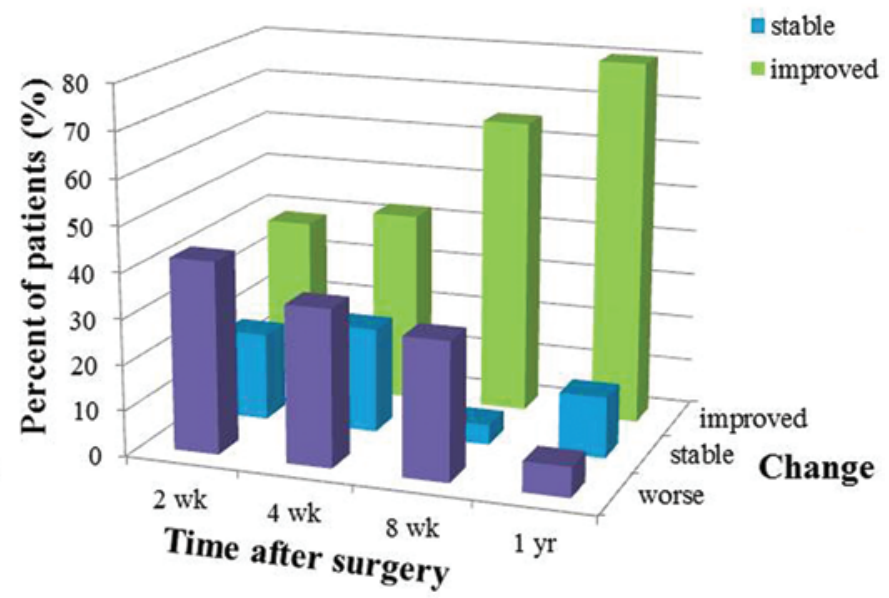

FIG. 4. Percent of patients with changes in headache postoperatively in relation to baseline scores. Three-dimensional bar graphs show the percent of patients with better, stable, or worse headaches after surgery in endoscopic patients (left) and microscopic patients (right). Endoscopic and microscopic patients show similar headache improvement. By 1 year, $90 \%$ of endoscopic alone, microscopic alone, or microscopic and endoscopic patients analyzed together experience either an improvement beyond their baseline condition $(80 \%)$ or a return to their baseline status $(10 \%)$. Figure is available in color online only. 


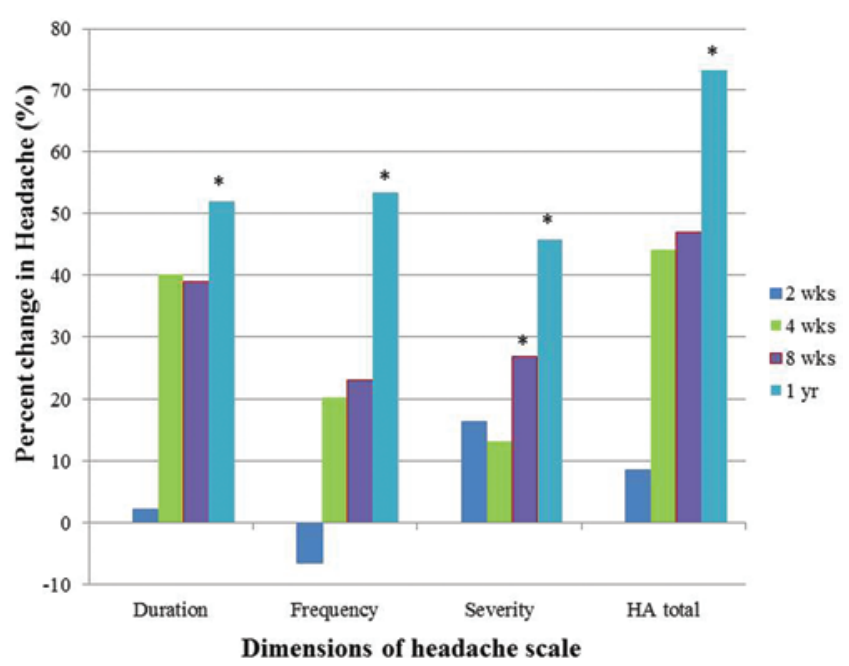

FIG. 5. Mean percent change in headache $(\mathrm{HA})$ scores from before to after surgery. Bar graph shows significant improvements in headache severity by 8 weeks postoperatively compared with baseline measures $(27 \%, p=0.028)$. Significant improvements are seen from before surgery to 1 year after in all dimensions of headache (total result 73\%, $p=$ 0.004 ; duration $52 \%, p=0.011$; severity $46 \%, p<0.001$; frequency $53 \%$, $p<0.001)$. Asterisk indicates a significant $(p<0.05)$ difference in mean headache scores. Figure is available in color online only.

weeks to 1 year after surgery. Overall, $80 \%$ of patients with headaches prior to surgery in the endoscopic and microscopic groups alone or as a combined group showed improvement by 1 year after surgery.

Baseline headache scores were not significantly different between microscopic and endoscopic patients, and postoperative scores at 2 and 4 weeks after treatment remained similar. By 8 weeks and 1 year after surgery no significant differences were found in headache duration, headache frequency, or total headache score. And while both endoscopic and microscopic patients experienced improvement in headache severity at 8 weeks and 1 year after treatment compared with the preoperative state, headache severity was significantly less in endoscopic than in microscopic patients ( 8 weeks: mean endoscopic $2.67 \mathrm{vs} \mathrm{mi-}$ croscopic $4.75, \mathrm{p}=0.026$; 1 year: mean endoscopic $2.10 \mathrm{vs}$ microscopic 3.87, $\mathrm{p}=0.045$ ).

Because of a lack of significant differences between microscopic and endoscopic groups, we decided to analyze both groups together to assess the change in postoperative headache scores compared with baseline. We did not observe significant changes at 2 or 4 weeks after surgery; however, by 8 weeks postoperatively, there were significant improvements in severity by $27 \%(\mathrm{p}=0.028$; Fig. 5). By 1 year, we observed significant improvements in all headache dimensions: total result $73 \%, \mathrm{p}=0.004$; duration $52 \%, \mathrm{p}=0.011$; severity $46 \%, \mathrm{p}<0.001$; frequency $53 \%$, $\mathrm{p}<0.001$.

\section{SF-36 Results}

Seventy-four patients completed the SF-36 (40 endoscopic and 34 microscopic). Compared with US population norms (raw score of 50 on each scale) both groups reported better than average functioning on all scales

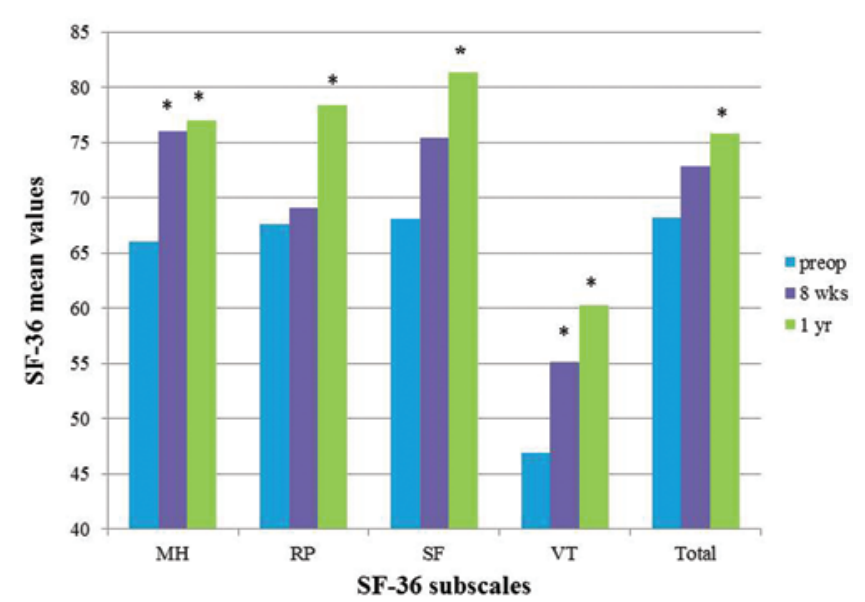

FIG. 6. Mean SF-36 domain values before and after surgery. No significant differences occurred between microscopic and endoscopic patients on total SF-36 or any of the 8 domains. Bar graph shows significant postoperative improvements compared with baseline measurements for the total SF-36 score and 4 of its 8 domains (only items with significant results are shown). By 8 weeks postoperatively, patients show significant improvement in mental health $(\mathrm{MH} ; 13 \%, p=0.005)$ and vitality (VT; $15 \%, p=0.037$ ) compared with preoperative baseline measures. By 1 year, this improvement expands to include $\mathrm{MH}(14 \%, p=0.03)$, role physical (RP; $14 \%, p=0.036$ ), social functioning (SF; $16 \%, p=0.009$ ), vitality (VT; $22 \%, p=0.002)$, and SF-36 total $(10 \%, p=0.024)$ compared with the preoperative state. Asterisk indicates a significant $(p<0.05)$ difference in mean SF-36 scores from baseline. Figure is available in color online only

except for vitality at baseline preoperative measurements (average endoscopic vitality $=45.63$, average microscopic vitality $=48.35$, combined average of vitality $=46.88$ ).

There were no significant improvements in the SF-36 in the microscopic group at the 8-week or 1-year time points. Endoscopic patients experienced significant improvements 8 weeks after surgery compared with baseline measurements (mental health $17 \%, \mathrm{p}=0.003$; vitality $20 \%$, $\mathrm{p}=0.031$ ). By 1 year after treatment, the endoscopic group exhibited significant improvements in mental health (17\%, $\mathrm{p}=0.009)$, social functioning $(22 \%, \mathrm{p}=0.005)$, vitality $(29 \%, \mathrm{p}=0.003)$, and total SF-36 (13\%, $\mathrm{p}=0.033)$.

There were no significant differences at any time point between the microscopic and endoscopic groups for the total SF-36 or for any of the 8 subscales. Consequently we decided to analyze endoscopic and microscopic cohorts as 1 group to compare the follow-up scores to baseline measures. Patients experienced improvement in all domains of the SF-36 at 8 weeks and 1 year postoperatively (Fig. 6). Specifically, the 8-week mark showed significant improvement in mental health $(13 \%, \mathrm{p}=0.005)$ and vitality $(15 \%$, $\mathrm{p}=0.037)$. By 1 year, this improvement expanded to include mental health $(14 \%, \mathrm{p}=0.03)$, role physical $(14 \%, \mathrm{p}=$ $0.036)$, social functioning $(16 \%, \mathrm{p}=0.009)$, vitality $(22 \%$, $\mathrm{p}=0.002)$, and SF-36 total $(10 \%, \mathrm{p}=0.024)$.

\section{Discussion}

This study prospectively compared the sinonasal, headache, and QOL outcomes of the microscopic and endoscopic approaches at a single institution during a concur- 
rent time period using the same perioperative care. Our findings suggest that the overall time course of recovery is similar for patients undergoing both techniques. However, our study also finds that patients undergoing an endoscopic approach experience improvement sooner after surgery since they displayed fewer nasal symptoms in the early phase of recovery.

\section{Nasal and Nasal-Related QOL Progression}

Our results indicate patients in both the microscopic and endoscopic groups are most symptomatic for nasal, facial, and psychological distress at 2 weeks after transsphenoidal surgery. Sleep was the only exception, which we found to be worse in the first 2 days after surgery. Confounding factors such as hospital personnel waking the patient up for tests and clinical assessments may have affected the sleep results during the perioperative time period. A Cornell group reported a similar recovery curve for patients who underwent endoscopic surgery, with patients showing a decline in nasal function at 3 weeks postoperatively. ${ }^{20}$ However, in that study, nonpituitary cases composed approximately $30 \%$ of that cohort. Another study comparing nasal function in nonpituitary and pituitary cases after endoscopic skull base surgery found no significant decline in nasal function at 3 weeks after surgery. ${ }^{2}$ Our study was limited to pituitary patients with the same pathology and investigated at 2 earlier postoperative time points (at 2448 hours and 2 weeks postoperatively), allowing a more detailed analysis of early postoperative nasal symptoms. We also saw an improvement in our patients' nasal condition as assessed by SNOT-20 and NOSE scores at 8 weeks after surgery. Similar studies have indicated a trend of improvement that reached significance at 3 months $^{13}$ and 6 months. ${ }^{2}$ Additionally, both of our surgical groups displayed improvement in their overall QOL as seen by higher SF-36 and SNOT-20 QOL scores. These results agree with previous research showing a return to baseline by 6 and 12 weeks and a significant improvement in QOL by 6 and 12 months $^{20}$ and between 18 months and 49 months. ${ }^{12}$

Recent studies have focused on the nasal symptoms and QOL for patients who undergo endoscopic transsphenoidal surgery; however, no study has made a comparison with patients who undergo a microscopic approach at the same institution during a concurrent time period. A few studies have considered the differences in patient experience for each approach. ${ }^{12,19}$

Graham and colleagues sent SNOT-22 questionnaires (similar to the SNOT-20 with the addition of 2 dimensions: sense of taste/smell and blockage/congestion of nose) to patients who had previously undergone either endoscopic or microscopic transsphenoidal surgery for a variety of parasellar pathologies. ${ }^{12}$ However, SNOT-22 scores were not attained at uniform times after surgery; the average time from surgery was 18.8 months for the endoscopic group and 49.3 months for the microscopic group. The authors reported that patients in the endoscopic group had better total scores and lower rhinology-specific scores than those in the microscopic group. However, Lwu and colleagues found conflicting results. ${ }^{19}$ Using an interview-based retrospective study to gather information about postsurgical satisfaction from patients who had undergone both a prior endoscopic and a separate microscopic transsphenoidal procedure for various parasellar pathologies, those authors concluded that patients favored endoscopic surgery over microscopic surgery despite a lack of rhinologic symptomatic differences or other complications in the recovery.

We find that the largest differences in microscopic and endoscopic postoperative experiences occur during the perioperative period, with patients in the endoscopic group reporting fewer rhinologic symptoms, less ear and face pain, and fewer sleep disturbances. These results are interesting compared with Lwu's qualitative patient interviews. One possible interpretation is that while patients do experience significantly more rhinologic symptoms up to 2 weeks after surgery when assessed with a quantitative survey, patients interviewed retrospectively may not remember their experience well enough to accurately describe their symptoms.

Other considerations for differences between microscopic and endoscopic nasal symptoms include possible CSF leak, nasal packing, and sinonasal swelling. Cerebrospinal fluid leakage was not a factor in our study since no patients experienced a leak postoperatively. A greater frequency of nasal and sleep problems in patients who underwent microscopic surgery may have been due to the presence and removal of nasal packing (not part of the endoscopic protocol) within the first 24 hours after surgery, which can create nasal irritation and disrupt sleep. A somewhat surprising result is the significantly worse nasal symptoms at 8 weeks postoperatively with the microscopic technique compared with the endoscopic technique. One possible explanation may be that the swelling that occurs between 2 and 4 weeks after surgery may be similar for both groups, but that microscopic patients may require a slightly longer time to recover. The result is that endoscopic cases experience a faster recovery rate, which becomes more equal between the 2 groups by the 1 -year follow-up. Both groups continue to experience an improvement in symptoms at 8 weeks and beyond, however.

Compared with previous studies, ${ }^{12,19}$ our study analyzes specific early and late time periods after transsphenoidal surgery for one specific diagnosis, NFAs. The benefit of surveying patients at multiple time points is that it allows us to gather data on the fine details of patient recovery. Unlike Graham and colleagues, who concluded that endoscopic patients surveyed at about 1.5 years had better outcomes than microscopic patients surveyed at about 4 years postoperatively, ${ }^{12}$ our data suggest that patients in both groups have a similar resolution of symptoms and QOL by 1 year after surgery. Additionally, our results agree with the long-term rhinonasal outcomes reported by Lwu and colleagues, who used the open-ended interview question study ${ }^{19}$ and add a more detailed picture of what happens in the early recovery stages. We report that microscopic and endoscopic groups experience a return to baseline by 1 year following surgery and an improvement in sinonasal function after transsphenoidal surgery. These data align with previous findings suggesting that patients with pituitary adenomas experience decreased QOL before surgery compared with population norms. ${ }^{16}$ Patients also experience an improvement in sinonasal functioning, sleep, and psychological impairments on the SNOT-20 and NOSE 
scales at 1 year after treatment compared with baseline. We hypothesize that the patients' sinonasal symptoms improve, in part, because of the increased nasal care and education provided in the postoperative period regarding the importance of nasal rinses and nasal sprays on recovery. In addition, both operative approaches allow repair of septal deviation and outfracturing of the nasal turbinates.

Patients often express concern over a loss of smell because of transsphenoidal surgery. Further study using the SNOT-22 and the full version of the NOSE, which include questions concerning the sense of smell, would create a more accurate assessment of the differences between microscopic and endoscopic techniques. In a study utilizing the sense of smell, Kahilogullari and colleagues found, through the use of the Smell Diskettes Olfaction Test, that the endoscope was more effective than the microscope in preserving olfactory function up to 6 months after surgery. ${ }^{17}$ Although these added items would affect the total of the SNOT and NOSE construct scores, the addition of olfactory items would probably not affect the overall trend of our data given our specific analysis of the individual NOSE components and SNOT-20 subscales.

\section{Headache Progression}

Headache is frequently a primary symptom of pituitary adenomas, particularly in macroadenomas or secreting adenomas. Prior studies have indicated a $62 \%^{5}$ to $77 \% \%^{11}$ incidence of headache in patients with NFAs and as low as $28 \%{ }^{14}$ when headache is defined as a primary symptom. Our study found headache in $70 \%$ of patients $(67 \%$ of endoscopic and $75 \%$ of microscopic) who had presented with an NFA for surgical treatment.

Prior studies have shown improvement in $90 \%$ of patients with a nonfunctioning microadenoma who reported standard prophylaxis-resistant headache as a presenting complaint. ${ }^{10}$ Another study reported headache improvement in $11 \%$ of patients at a 6-week follow-up and improvement in 53\% of patients at a 6-month follow-up among patients who had transsphenoidal surgery for pituitary adenoma.${ }^{14}$ Our study found that headaches improved in $51 \%$ of patients at 2 weeks, $68 \%$ of patients at 4 weeks, and $70 \%$ of patients at 8 weeks postoperatively. Additionally we found that $80 \%$ of patients in the microscopic or endoscopic group alone or in the 2 groups combined experienced improved headache or resolution of symptoms by 1 year postoperatively. When microscopic and endoscopic cases were combined, significant improvements were found in all headache dimensions, including total headache result, duration, frequency, and severity in relation to the preoperative state, by 1 year after surgery.

Explanations for preoperative and postoperative headache related to pituitary adenomas are unclear. Preoperatively, headaches may be caused by stretching of the diaphragma sellae, but new evidence suggests that tumor size is not a great predictor of headache levels. ${ }^{14}$ Some patients with small tumors report significant headache, whereas some with large tumors report none.

Headaches after surgery may relate to a number of factors. Early after surgery, pain is probably related to trauma to the nasal mucosa, resection of the bony septum, and resection of bone of the sphenoid. Pain can also be due to nasal congestion and sinus pressure, particularly during the weeks to month after surgery. Our results suggest that while headaches begin to improve at 4 and 8 weeks after surgery, they do not significantly improve until 1 year postoperatively. The only significant difference we saw in the endoscopic and microscopic patients was in headache severity at 8 weeks and 1 year postoperatively. Microscopic patients may require longer healing times than endoscopic patients, and their headaches may remain longer. However, headache severity differences between the 2 techniques seen at 1 year are unlikely to be related to continued healing, and further explanation cannot be established at this time.

Our data probably underestimate the extent of improvement in headaches postoperatively since all levels-very mild or extensive-of duration, frequency, and severity were included under the definition of having headache. We did not distinguish between patients who presented with a primary complaint of headache and those who reported any type of headache. By analyzing only patients with primary headache, we suspect more significant changes would have been seen earlier in the recovery process. Earlier data suggest that recovery from headache is more rapid in patients who report headache as a chief complaint as opposed to a secondary complaint. ${ }^{14}$ However, these data have also shown that the improvement rate at 6 months was comparable in the 2 headache groups. Therefore, we are confident in our headache results at 8 weeks and 1 year regardless of the primary or secondary preoperative headache classification.

These results are important in patient care in a variety of ways. First, our data indicate that the majority of patients will experience significant improvements in headache dimensions by 1 year after either surgical technique. Secondly, our data suggest that a subset of patients may initially experience a worsening of headache symptoms prior to long-term improvement. Finally, our data indicate no substantial difference in headache improvement between microscopic and endoscopic techniques.

\section{SF-36 Progression}

Patients with pituitary adenomas report diminished QOL both before ${ }^{16,28}$ and after treatment. ${ }^{7,28,29}$ Factors related to a decrease in self-reported QOL include radiotherapy, pituitary surgery, and pituitary deficiencies as well as disease-specific effects of having a pituitary adenoma. Studies have clearly documented hypersecreting disease-specific impairments, particularly in acromegaly and Cushing's patients. ${ }^{16,29}$ Patients with acromegaly experience impaired physical function, whereas patients with Cushing's disease report severe impairment on all measures compared with patients with other pituitary adenoma diagnoses in both short-term and long-term (average 13 years) studies. However, only a few studies have focused specifically on NFAs with somewhat conflicting results.

One of the original studies to assess QOL measures (SF-36) in patients with untreated pituitary adenomas compared NFA patients against both US population norms and other disease-specific pituitary adenoma diagnoses. ${ }^{16}$ In this study, Johnson et al. reported that NFA patients experienced QOL measures significantly below the US 
normative values in all subscales of the SF-36. The lowest of these was in the area of vitality (followed by role emotional, social function, role physical, and mental health). A UK study by Capatina and colleagues reported similar low levels of vitality after surgery, as well as in NFA patients who took the SF-36 an average of 72 months after diagnosis. ${ }^{4}$

Our study confirms that NFA patients experience a substantial decrease in vitality compared with the US population norms prior to surgery and continue to experience low levels of vitality after surgery. Conversely we report scores above population norms in all other dimensions of the SF36. Two factors may account for this difference. First, our study included all NFAs of any size, whereas the study by Johnson and associates ${ }^{16}$ included only patients with macroadenomas. Including only macroadenomas may have increased the visual, pain, and hormonal abnormalities in their sample, leading to lower preoperative QOL scores. Second, our study was conducted a decade or more later; therefore, our patients may have been exposed to newer, more effective treatments earlier in the disease process. Our results concur with those in subsequent studies that report a decrease in preoperative scores compared with age-matched controls, but this decrease was not lower than US normative values.

Other studies have attempted to compare QOL in NFA patients with that in patients with other medical illnesses. In a study comparing NFA to an illness requiring similar surgery, Page et al. did not find reduced functioning in treated NFA patients compared with functioning in patients after mastoid surgery, pointing toward a general influence of medical illness on QOL. ${ }^{25}$ According to Dekkers et al., in comparison with age-matched healthy controls, NFA patients report increased mental and physical fatigue, reduced energy, physical role limitations due to reduced motivation, physical problems, and activity as well as emotional problems.?

The above-mentioned studies drew comparisons from 3 very different populations. Dekkers et al. used average scores from healthy controls ranging from 70 to $93,{ }^{7}$ whereas Johnson et al. used US population norms set at $50 .{ }^{16}$ Page et al. reported a general influence of medical illness on QOL, with scores ranging from 71 to $83 .{ }^{25}$ While these discrepancies in comparison values lead to difficulties in drawing conclusions about comparisons between populations, we present a unifying conclusion. Our data most closely match those by Page et al., indicating that patients experience a decline in QOL in relation to healthy controls comparable to other similar medical illnesses before and after surgery. Additionally, we report that patients experience baseline levels of vitality that are below US population norms.

Recent studies have taken a prospective approach to determine if patients improve in QOL after surgery compared with self-reported preoperative measures. McCoul et al. found significant improvements in performance, vitality, physical function, pain, and emotional impact at 12 weeks and 6 months after surgery in patients with skull base tumors, with no significant decline or improvement at 3 or 6 weeks after surgery. ${ }^{21}$ Tanemura et al. concluded that patients experience a decrease in physical scores 1 month after the operation while experiencing an increase in mental scores. ${ }^{28}$ They further reported that by 6 months patients recover to the level of the normal population in physical scores and remain stable in increased mental scores. Both of these studies were conducted on patients who underwent a pure endoscopic transsphenoidal approach.

Our data extend these findings to 1 year after surgery and also evaluate the QOL for patients who receive microscopic treatment. Our results indicate that both endoscopic and microscopic patients experience an improvement in QOL by 1 year, with no significant differences between the 2 techniques. However, our patients showed SF-36 scores that were lower than the normal controls used in the Dekker et al. study. This suggests that while our patients did improve in QOL by 1 year after surgery, they may still be functioning at a level that is lower than healthy controls. Confounding variables were not noted; however, throughout the year it is possible that patients experience life changes other than surgical recovery that influence the QOL scores. A larger sample size would be helpful to minimize such effects.

Vitality, social function, mental health, and ability to perform work roles are 4 areas in which patients experienced lower preoperative functioning that improved significantly after surgery. These dimensions of the SF-36 have been found to be important for well-being in a variety of ways. Reduced levels of vitality (that is, low energy level and fatigue) and reduced social functioning have been used to accurately distinguish depressed from nondepressed individuals. ${ }^{6}$ Fatigue has been shown to be both predictive of and a consequence of depression. ${ }^{1}$ Positive social relations are predictors of happiness, ${ }^{9}$ while social connectedness is a primary predictor of resilience in people who experience high stress, and lack of social connectedness is integral to the development of posttraumatic stress disorders. ${ }^{22}$ Lastly, work performance is associated with perceived health and vitality. ${ }^{30}$ Therefore, increased vitality, social functioning, mental health, and ability to perform work roles are all essential to QOL and an important benefit of surgical treatment for NFAs.

Our results indicate patients with NFAs improve significantly beyond their baseline levels within 1 year of surgery, and outcomes do not differ based on the technique used. More research in the area of disease-specific long-term QOL after treatment for pituitary adenomas is necessary to present patients with appropriate short- and long-term expectations.

\section{Study Advantages and Limitations}

Our study was conducted at one institution and therefore eliminated confounding variables of differences in personnel, hospital procedures, or treatment plans across various institutions. Patients were given surveys using a standard protocol by a single coordinator in approximately $90 \%$ of the sample. Remaining surveys were distributed by mail or given by a different coordinator. Additionally, we chose a timeline of follow-up measurements to capture acute, short-term, and long-term symptoms, which allowed us to capture subtle changes that may occur at early and later stages of recovery. 
The primary limitation of our study is the consistency with which patients participated in the follow-up surveys at each time point. Ideally, each patient would have received an opportunity to complete the survey at each time point and returned the completed surveys; however, patient compliance and logistical procedures resulted in some loss of completed survey data. Surveys performed at 2 and 4 weeks after surgery were the time points most affected by a decrease in collected data. Additionally, the questionnaires at 2 and 4 weeks were completed by telephone, which may have led to a different response than the paper-based surveys. Nonetheless, our total sample size at all time points is similar to those in other studies that have measured QOL, ${ }^{19}$ headache, ${ }^{18}$ and sinonasal function ${ }^{17,20,27}$ after transsphenoidal surgery. Additionally, the sample size is similar to those in other studies that have provided comparative analyses of $\mathrm{QOL}^{19}$ and nasal function ${ }^{17}$ between microscopic and endoscopic techniques.

\section{Conclusions}

Our study indicates that in the early stages of postoperative recovery, patients who undergo the endoscopic procedure have significantly fewer rhinologic symptoms than patients who undergo the microscopic procedure. The postsurgical state at 2 weeks is the most symptomatic for patients who undergo either technique. Long-term QOL, headache pain, and nasal outcome improve beyond baseline levels following both procedures and are significantly improved at 1 year after surgery in terms of headache score (including duration, severity, and frequency), NOSE score, and SF-36 score (including mental health, physical work roles, vitality, and social functioning). However, the SF-36 scores remain lower than reported controls, particularly in levels of vitality. This information may be useful before and after surgery when counseling patients concerning symptoms and outcomes related to specific microscopic or endoscopic techniques.

\section{References}

1. Addington J, Addington D, Gasbarre L: Neurocognitive and social functioning in schizophrenia and other diagnoses. Schizophr Res 48:367-368, 2001

2. Bedrosian JC, McCoul ED, Raithatha R, Akselrod OA, Anand VK, Schwartz TH: A prospective study of postoperative symptoms in sinonasal quality-of-life following endoscopic skull-base surgery: dissociations based on specific symptoms. Int Forum Allergy Rhinol 3:664-669, 2013

3. Browne JP, Hopkins C, Slack R, Cano SJ: The Sino-Nasal Outcome Test (SNOT): can we make it more clinically meaningful? Otolaryngol Head Neck Surg 136:736-741, 2007

4. Capatina C, Christodoulides C, Fernandez A, Cudlip S, Grossman AB, Wass JA, et al: Current treatment protocols can offer a normal or near-normal quality of life in the majority of patients with non-functioning pituitary adenomas. Clin Endocrinol (Oxf) 78:86-93, 2013

5. Chen L, White WL, Spetzler RF, Xu B: A prospective study of nonfunctioning pituitary adenomas: presentation, management, and clinical outcome. J Neurooncol 102:129-138, 2011

6. Christensen L, Duncan K: Distinguishing depressed from nondepressed individuals using energy and psychosocial variables. J Consult Clin Psychol 63:495-498, 1995

7. Dekkers OM, van der Klaauw AA, Pereira AM, Biermasz
NR, Honkoop PJ, Roelfsema F, et al: Quality of life is decreased after treatment for nonfunctioning pituitary macroadenoma. J Clin Endocrinol Metab 91:3364-3369, 2006

8. D'Haens J, Van Rompaey K, Stadnik T, Haentjens P, Poppe $\mathrm{K}$, Velkeniers B: Fully endoscopic transsphenoidal surgery for functioning pituitary adenomas: a retrospective comparison with traditional transsphenoidal microsurgery in the same institution. Surg Neurol 72:336-340, 2009

9. Diener E, Seligman ME: Very happy people. Psychol Sci 13:81-84, 2002

10. Fleseriu M, Yedinak C, Campbell C, Delashaw JB: Significant headache improvement after transsphenoidal surgery in patients with small sellar lesions. J Neurosurg 110:354-358, 2009

11. Gondim JA, de Almeida JP, de Albuquerque LA, Schops M, Gomes E, Ferraz T: Headache associated with pituitary tumors. J Headache Pain 10:15-20, 2009

12. Graham SM, Iseli TA, Karnell LH, Clinger JD, Hitchon PW, Greenlee JD: Endoscopic approach for pituitary surgery improves rhinologic outcomes. Ann Otol Rhinol Laryngol 118:630-635, 2009

13. Harrow BR, Batra PS: Sinonasal quality of life outcomes after minimally invasive resection of sinonasal and skull-base tumors. Int Forum Allergy Rhinol 3:1013-1020, 2013

14. Jahangiri A, Wagner JR, Chin AT, Han SW, Tran MT, Miller LM, et al: Incidence of headache as a presenting complaint in over 1000 patients with sellar lesions and factors predicting postoperative improvement. Clin Neurol Neurosurg 132:16-20, 2015

15. Jane JA Jr, Han J, Prevedello DM, Jagannathan J, Dumont AS, Laws ER Jr: Perspectives on endoscopic transsphenoidal surgery. Neurosurg Focus 19(6):E2, 2005

16. Johnson MD, Woodburn CJ, Vance ML: Quality of life in patients with a pituitary adenoma. Pituitary 6:81-87, 2003

17. Kahilogullari G, Beton S, Al-Beyati ES, Kantarcioglu O, Bozkurt M, Kantarcioglu E, et al: Olfactory functions after transsphenoidal pituitary surgery: endoscopic versus microscopic approach. Laryngoscope 123:2112-2119, 2013

18. Levy MJ, Jäger HR, Powell M, Matharu MS, Meeran K, Goadsby PJ: Pituitary volume and headache: size is not everything. Arch Neurol 61:721-725, 2004

19. Lwu S, Edem I, Banton B, Bernstein M, Vescan A, Gentili F, et al: Quality of life after transsphenoidal pituitary surgery: a qualitative study. Acta Neurochir (Wien) 154:1917-1922, 2012

20. McCoul ED, Anand VK, Bedrosian JC, Schwartz TH: Endoscopic skull base surgery and its impact on sinonasal-related quality of life. Int Forum Allergy Rhinol 2:174-181, 2012

21. McCoul ED, Anand VK, Schwartz TH: Improvements in site-specific quality of life 6 months after endoscopic anterior skull base surgery: a prospective study. J Neurosurg 117:498-506, 2012

22. McDermott B, Berry H, Cobham V: Social connectedness: a potential aetiological factor in the development of child posttraumatic stress disorder. Aust N Z J Psychiatry 46:109-117, 2012

23. Oldfield EH, Jane JA Jr: Endoscopic versus microscopic pituitary surgery. J Neurol Neurosurg Psychiatry 84:827, 2013

24. Oldfield EH, Vortmeyer AO: Development of a histological pseudocapsule and its use as a surgical capsule in the excision of pituitary tumors. J Neurosurg 104:7-19, 2006

25. Page RC, Hammersley MS, Burke CW, Wass JA: An account of the quality of life of patients after treatment for non-functioning pituitary tumours. Clin Endocrinol (Oxf) 46:401-406, 1997

26. Piccirillo JF, Merritt MG Jr, Richards ML: Psychometric and clinimetric validity of the 20-Item Sino-Nasal Outcome Test (SNOT-20). Otolaryngol Head Neck Surg 126:41-47, 2002

27. Stewart MG, Smith TL, Weaver EM, Witsell DL, Yueh B, 
Hannley MT, et al: Outcomes after nasal septoplasty: results from the Nasal Obstruction Septoplasty Effectiveness (NOSE) study. Otolaryngol Head Neck Surg 130:283-290, 2004

28. Tanemura E, Nagatani T, Aimi Y, Kishida Y, Takeuchi K, Wakabayashi T: Quality of life in nonfunctioning pituitary macroadenoma patients before and after surgical treatment. Acta Neurochir (Wien) 154:1895-1902, 2012

29. van der Klaauw AA, Kars M, Biermasz NR, Roelfsema F, Dekkers OM, Corssmit EP, et al: Disease-specific impairments in quality of life during long-term follow-up of patients with different pituitary adenomas. Clin Endocrinol (Oxf) 69:775-784, 2008

30. van Scheppingen AR, de Vroome EMM, ten Have KC, Zwetsloot GI, Bos EH, van Mechelen W: Motivations for health and their associations with lifestyle, work style, health, vitality, and employee productivity. J Occup Environ Med 56:540-546, 2014

31. Ware JE Jr, Sherbourne CD: The MOS 36-item short-form health survey (SF-36). I. Conceptual framework and item selection. Med Care 30:473-483, 1992

\section{Disclosures}

The authors report no conflict of interest concerning the materials or methods used in this study or the findings specified in this paper.

\section{Author Contributions}

Conception and design: Jane, Elzoghby. Acquisition of data: Pledger, Elzoghby. Analysis and interpretation of data: Pledger. Drafting the article: Pledger. Critically revising the article: Jane, Oldfield, Payne. Reviewed submitted version of manuscript: Jane. Approved the final version of the manuscript on behalf of all authors: Jane. Administrative/technical/material support: Pledger. Study supervision: Jane.

\section{Correspondence}

John A. Jane Jr., Department of Neurosurgery, University of Virginia Health System, Box 800212, Charlottesville, VA 22908. email: jaj2k@virginia.edu. 